\title{
Tiny Obturator Node Metastasis from Prostate Cancer Not Shown by FDG-PET/CT, CT, or MRI Detected by ${ }^{11} \mathrm{C}$-Choline PET/CT
}

\author{
Kazuhiro Kitajima $^{a}$ Shingo Yamamoto ${ }^{b}$ Soichi Odawara ${ }^{c}$ \\ Yusuke Kawanaka ${ }^{c}$ Yukako Nakanishi ${ }^{b}$ Takahiko Hashimoto $^{b}$ \\ Yusuke Yamada $^{\mathrm{b}}$ Toru Suzuki $^{\mathrm{b}}$ Akihiro Kanematsu ${ }^{\mathrm{b}}$ Michio Nojima ${ }^{\mathrm{b}}$ \\ Neinei Kimura $^{d}$ Masataka Zouzumi $^{d}$ Seiichi Hirota $^{d}$ \\ Koichiro Yamakadoc \\ ${ }^{a}$ Department of Radiology, Division of Nuclear Medicine and PET Center, Hyogo College \\ of Medicine, Nishinomiya, Japan; ${ }^{b}$ Department of Urology, Hyogo College of Medicine, \\ Nishinomiya, Japan; 'Department of Radiology, Hyogo College of Medicine, \\ Nishinomiya, Japan; ${ }^{d}$ Department of Surgical Pathology, Hyogo College of Medicine, \\ Nishinomiya, Japan
}

\section{Keywords}

Choline · FDG · PET · Lymph node metastasis · Prostate cancer

\begin{abstract}
We report a 65-year-old male with histopathologically proven prostate cancer and multiple pelvic node metastases using a robotic-assisted radical prostatectomy procedure plus extended pelvic lymph node dissection. Positron emission tomography (PET) scan findings demonstrated a moderate accumulation of ${ }^{11} \mathrm{C}$-choline in a metastatic left obturator node sized $8 \times 8 \mathrm{~mm}$, though only a faint uptake of fluorodeoxyglucose (FDG) was noted. ${ }^{11} \mathrm{C}$ choline PET/computed tomography (CT) may be useful for the diagnosis of a tiny metastatic lymph node not demonstrated by $\mathrm{CT}$, magnetic resonance imaging, or FDG-PET/CT and to determine the need for an extended pelvic lymph node dissection.
\end{abstract}


 Oncology}

\section{Introduction}

Lymph node (LN) evaluation findings have important therapeutic and prognostic significance for prostate cancer ( $\mathrm{PCa}$ ) cases. In intermediate- and high-risk PCa patients undergoing a radical prostatectomy (RP), an extended pelvic lymph node dissection (ePLND) including the obturator fossa and internal, external, and common iliac fields is considered to be the gold standard procedure to determine LN status [1]. However, ePLND is invasive and time consuming and also associated with morbidity, thus is not warranted for all PCa cases, with a limited PLND generally preferred for patients at low risk of PCa. An accurate method for preoperative nodal staging at the time of initial diagnosis of PCa would be useful to guide treatment decisions, limit the extent of the PLND on an individual basis, and possibly even spare use of an invasive procedure such as an ePLND.

Along this line, the development of a sensitive and reliable noninvasive procedure for detecting nodal involvement is important. Conventional cross-sectional imaging techniques, such as computed tomography (CT) and magnetic resonance imaging (MRI), are not able to accurately diagnose nodal involvement in a case of PCa. In a meta-analysis conducted by Hövels et al. [2], a sensitivity of approximately $40 \%$ has been reported for both of those modalities. Furthermore, in a contemporary cohort of more than 1,500 PCa patients treated with RP and ePLND, an unsatisfyingly low sensitivity of only $13 \%$ was noted for preoperative detection of LN metastasis via CT [3]. The criteria for nodal characterization using CT or MRI findings are primarily reliant on morphologic assessment based on size and shape, with a nodal short axis diameter of $1 \mathrm{~cm}$ generally accepted as the upper limit of normal. However, size as a criterion does not seem sufficient to determine LN status in PCa patients, as it has been reported that in newly diagnosed PCa cases up to $80 \%$ of the instances of LN metastasis occur in normal-sized LNs $(<8 \mathrm{~mm})$ [4].

It has been speculated that these limitations might be overcome by molecular imaging techniques such as choline positron emission tomography (PET) in combination with CT (PET/CT) in PCa cases [5-7]. Here, we report a case in which PET/CT [11C]-labeled choline findings were useful to diagnose left obturator node metastasis $(8 \times 8 \mathrm{~mm})$ that was not revealed by fluorodeoxyglucose (FDG)-PET/CT, MRI, or CT. Furthermore, we present details of a guided ePLND procedure used to treat $\mathrm{PCa}$ in this patient.

\section{Case Report}

A 65-year-old male with biopsy-proven PCa, based on a serum prostate-specific antigen (PSA) level of $8.8 \mathrm{ng} / \mathrm{mL}$, came to our hospital for initial treatment. Diagnostic confirmation of PCa in the bilateral zone was achieved by standard transrectal 12-core biopsy findings (adenocarcinoma, Gleason score $5+4$ ), which indicated this to be a high-risk case. Preoperative contrast-enhanced CT (Fig. 1a) and pelvic MRI (Fig. 1b) results showed mild swelling of the left obturator LN, which measured $8 \times 8 \mathrm{~mm}$. Pelvic MRI showed multiple hypointense areas in the bilateral prostate gland on T2-weighted images. Whole-body FDG-PET/CT scanning demonstrated mild FDG uptake in the left prostate zone, with faint FDG uptake with a maximum standardized uptake value (SUVmax) of 1.95 in the left obturator node (Fig. 1c). Subsequently, ${ }^{11} \mathrm{C}$-choline PET/CT scanning showed a strong accumulation of ${ }^{11} \mathrm{C}$-choline in the bilateral prostate gland and a moderate uptake of ${ }^{11} \mathrm{C}$-choline with an SUVmax value of 4.16 in the left obturator node (Fig. 1d). The left obturator node was suspected to be metastatic based on the ${ }^{11} \mathrm{C}$-choline PET/CT results. Clinical stage was T2bN1M0. 
Physicians at the Department of Urology performed a robotic-assisted RP procedure plus ePLND, which included internal, external, and obturator LNs as well as common LNs up to the cross of the ureters. A pathologic examination revealed a multifocal Gleason score of $5+5$, pT3b PCa, with subtle extracapsular and seminal vesicle invasion. Pathological analysis of the LNs showed that 4 of 25 left obturator fossa LNs (Fig. 2), 2 of 13 left external iliac LNs, 1 of 5 left common iliac LNs, and 5 of 27 right obturator fossa LNs were metastatic. MRI, ${ }^{11} \mathrm{C}$-choline PET/CT, and FDG-PET/CT examinations were unable to detect subtle extracapsular or seminal vesicle invasion, or the 11 instances of tiny sub-centimeter LN metastasis other than the 8-mm left obturator node. The patient was scheduled to receive radiotherapy following surgery.

\section{Discussion}

PET/CT $\left[{ }^{11} \mathrm{C}\right]$ - and $\left[{ }^{18} \mathrm{~F}\right]$-labeled choline have been successfully used for restaging PCa patients with biochemical recurrence after undergoing definitive therapy, especially in cases with a PSA level $>1.0 \mathrm{ng} / \mathrm{mL}$ [8]. In selected patients with a high likelihood of regional or bone metastasis, pretreatment choline PET/CT is a useful noninvasive accurate staging tool. The most commonly used radiopharmaceutical agent for PET in the field of oncology is FDG, an analog of glucose that is preferentially taken up and trapped inside malignant cells. However, in urologic oncology cases, the use of FDG has been limited for the diagnosis of PCa, because of its low level of tumor uptake and urinary excretion. Furthermore, the superiority of choline PET/CT for staging and restaging PCa as compared with FDG-PET/CT has been demonstrated $[9,10]$.

Several groups have reported the diagnostic performance of PET/CT with ${ }^{11} \mathrm{C}$-choline or ${ }^{18} \mathrm{~F}-$ fluorocholine for the diagnosis of pelvic LN metastasis. In a report of 130 intermediateand high-risk PCa patients who underwent RP and ePLND with 912 LNs sampled, Beheshti et al. [5] noted that patient-based sensitivity and specificity for the detection of pelvic LN metastasis was 45 and 96\%, respectively. In the largest reported preoperative series of intermediate- and high-risk patients who underwent RP and ePLND $(n=210)$, Poulsen et al. [6] noted that patient-based sensitivity and specificity for the detection of pelvic LN metastasis were 73 and $88 \%$, respectively, with node-based sensitivity and specificity at 56 and $94 \%$, respectively.

Although choline PET/CT shows better performance than CT or MRI for the detection of locoregional LN metastasis, it does not provide an optimal detection rate in comparison with a lymphadenectomy and cannot replace that procedure. Contractor et al. [7] reported $28 \mathrm{PCa}$ patients who underwent preoperative ${ }^{11} \mathrm{C}$-choline PET/CT and MRI and demonstrated that sensitivity and specificity on a per nodal basis were 19 and 99\%, respectively, for MRI, and 52 and $98 \%$, respectively, for ${ }^{11} \mathrm{C}$-choline PET/CT. Two other groups reported that its sensitivity for small metastatic LN was low because of limited spatial resolution, with detection rates for metastatic LNs sized $<2,2-4.9,5-9.9$, and $>10 \mathrm{~mm}$ at $0,25-30,33-43$, and $77-90 \%$, respectively $[7,11]$.

\section{Conclusion}

In the present case, ${ }^{11} \mathrm{C}$-choline PET/CT was useful for the detection of an obturator node metastasis $8 \mathrm{~mm}$ in size that could not be diagnosed based on FDG-PET/CT, CT, or MRI 
findings. On the other hand, ${ }^{11} \mathrm{C}$-choline PET/CT was unable to detect 11 instances of subcentimeter node metastasis due to the low spatial resolution inherent with PET imaging, approximately $5 \mathrm{~mm}$ in clinical scanners. It is important to be aware of the advantages and disadvantages of ${ }^{11} \mathrm{C}$-choline PET/CT for imaging examinations of PCa patients.

\section{Statement of Ethics}

Informed consent was obtained from the patient for publication of this case report and any accompanying images. It is the policy of Hyogo College of Medicine that case reports do not require approval from an ethics review board or ethics committee.

\section{Disclosure Statement}

The authors declare that they have no conflicts of interest. We declare no financial support or relationship that may pose a conflict of interest.

\section{References}

1 Mottet N, Bellmunt J, Bolla M, Briers E, Cumberbatch MG, De Santis M et al. EAU-ESTRO-SIOG Guidelines on Prostate Cancer. Part 1: Screening, Diagnosis, and Local Treatment with Curative Intent. Eur Urol. 2017 Apr;71(4):618-29.

2 Hövels AM, Heesakkers RA, Adang EM, Jager GJ, Strum S, Hoogeveen YL et al. The diagnostic accuracy of CT and MRI in the staging of pelvic lymph nodes in patients with prostate cancer: a meta-analysis. Clin Radiol. 2008 Apr;63(4):387-95.

3 Briganti A, Abdollah F, Nini A, Suardi N, Gallina A, Capitanio U et al. Performance characteristics of computed tomography in detecting lymph node metastases in contemporary patients with prostate cancer treated with extended pelvic lymph node dissection. Eur Urol. 2012 Jun;61(6):1132-8.

4 Heesakkers RA, Hövels AM, Jager GJ, van den Bosch HC, Witjes JA, Raat HP et al. MRI with a lymph-nodespecific contrast agent as an alternative to CT scan and lymph-node dissection in patients with prostate cancer: a prospective multicohort study. Lancet Oncol. 2008 Sep;9(9):850-6.

5 Beheshti M, Imamovic L, Broinger G, Vali R, Waldenberger P, Stoiber F et al. 18F choline PET/CT in the preoperative staging of prostate cancer in patients with intermediate or high risk of extracapsular disease: a prospective study of 130 patients. Radiology. 2010 Mar;254(3):925-33.

6 Poulsen MH, Bouchelouche K, Hoilund-Carlsen PF, Petersen H, Gerke O, Steffansen SI et al. [18F]fluoromethylcholine (FCH) positron emission tomography/computed tomography (PET/CT) for lymph node staging of prostate cancer: a prospective study of 210 patients. BJU Int. 2012 Dec;110(11):1666-71.

7 Contractor K, Challapalli A, Barwick T, Winkler M, Hellawell G, Hazell S et al. Use of [11C]choline PET-CT as a noninvasive method for detecting pelvic lymph node status from prostate cancer and relationship with choline kinase expression. Clin Cancer Res. 2011 Dec;17(24):7673-83.

8 Kitajima K, Murphy RC, Nathan MA. Choline PET/CT for imaging prostate cancer: an update. Ann Nucl Med. 2013 Aug;27(7):581-91.

9 Beauregard JM, Williams SG, Degrado TR, Roselt P, Hicks RJ. Pilot comparison of F-fluorocholine and Ffluorodeoxyglucose PET/CT with conventional imaging in prostate cancer. J Med Imaging Radiat Oncol. 2010 Aug;54(4):325-32.

10 Richter JA, Rodríguez M, Rioja J, Peñuelas I, Martí-Climent J, Garrastachu P et al. Dual tracer 11C-choline and FDG-PET in the diagnosis of biochemical prostate cancer relapse after radical treatment. Mol Imaging Biol. 2010 Apr;12(2):210-7.

11 Schiavina R, Scattoni V, Castellucci P, Picchio M, Corti B, Briganti A et al. 11C-choline positron emission tomography/computerized tomography for preoperative lymph-node staging in intermediate-risk and highrisk prostate cancer: comparison with clinical staging nomograms. Eur Urol. 2008 Aug;54(2):392-401. 


\section{Case Reports in Oncology}

\begin{tabular}{l|l}
\hline Case Rep Oncol 2018;11:33-37 \\
\hline DOI: 10.1159/000486365 & $\begin{array}{l}\text { C 2018 The Author(s). Published by S. Karger AG, Basel } \\
\text { www.karger.com/cro }\end{array}$ \\
\hline
\end{tabular}

Kitajima et al: Tiny Obturator Node Metastasis from Prostate Cancer Not Shown by FDG-PET/CT, CT, or MRI Detected by ${ }^{11} \mathrm{C}$-Choline PET/CT
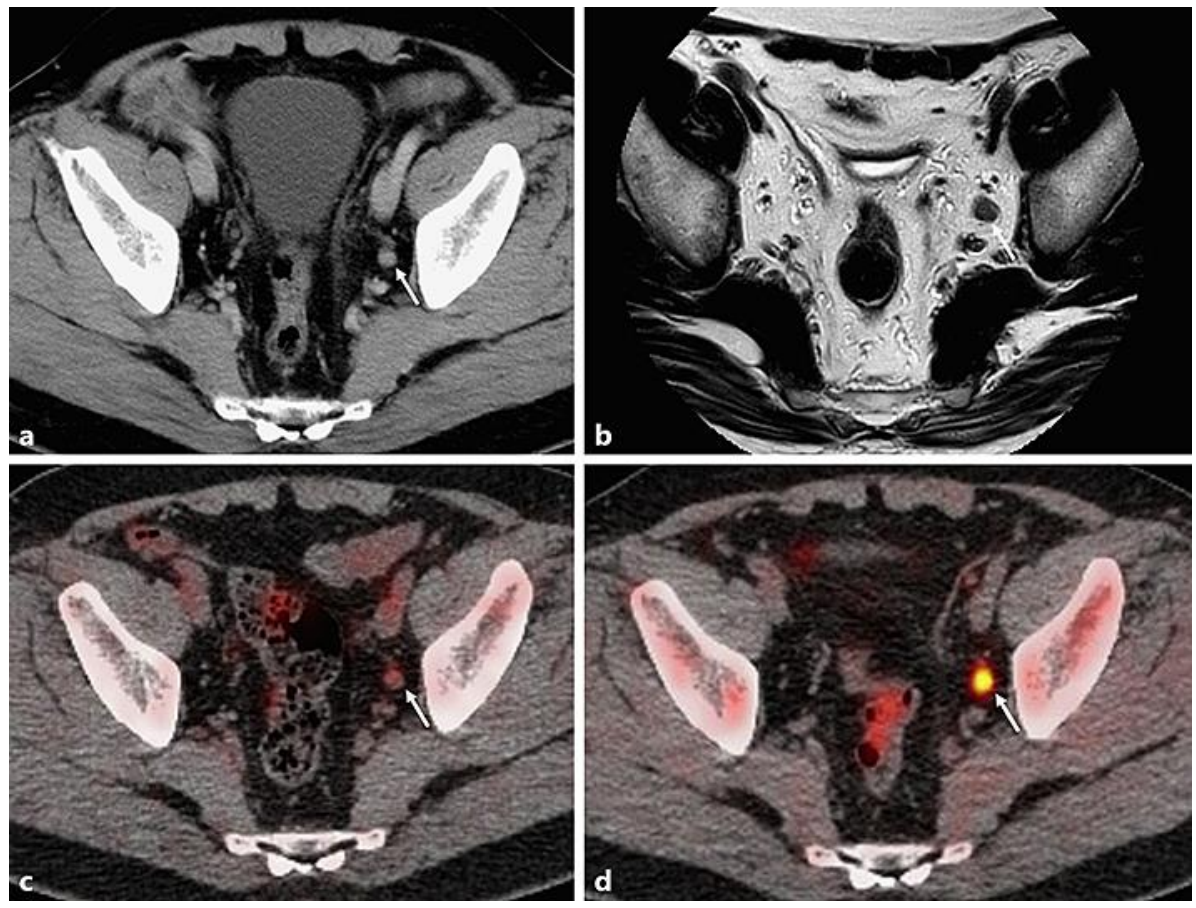

Fig. 1. A 65-year-old patient with pelvic node metastasis from prostate cancer. a Contrast-enhanced CT showing the left obturator node sized $8 \times 8 \mathrm{~mm}$ (arrow). b Pelvic T2-weighted MRI showing the left obturator node sized $8 \times 8 \mathrm{~mm}$ (arrow). c FDG-PET/CT showing faint FDG uptake (SUVmax: 1.95) in the 8-mm left obturator node (arrow). d ${ }^{11} \mathrm{C}$-choline PET/CT showing moderate ${ }^{11} \mathrm{C}$-choline uptake (SUVmax: 4.16) in the 8-mm left obturator node (arrow).

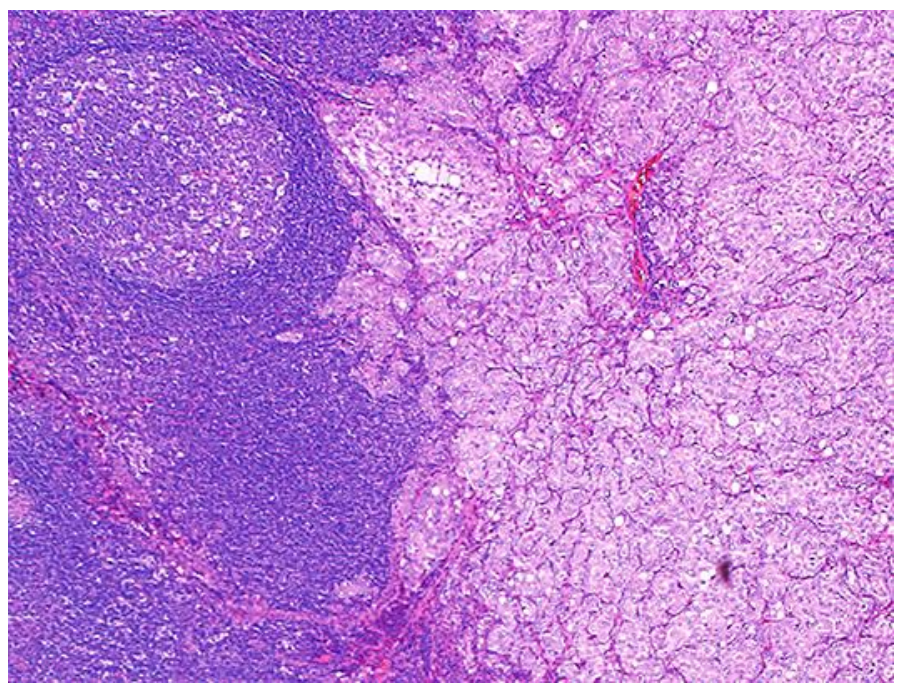

Fig. 2. Resected pelvic node metastasis. Histopathological findings of the left obturator node detected by ${ }^{11} \mathrm{C}$-choline PET/CT revealed invasion by prostate cancer cells. 